\title{
Electro-optic guest-host system containing azobenzene chromophores in PMMA matrix
}

\author{
A. Kozanecka-Szmigiel, ${ }^{* 1}$ K. Świtkowski ${ }^{1}$, B. Pura ${ }^{1}$, E. Schab-Balcerzak ${ }^{2}$, M. Siwy ${ }^{2}$ \\ ${ }^{I}$ Faculty of Physics, Warsaw University of Technology, Koszykowa 75, 00-662 Warszawa, \\ ${ }^{2}$ Centre of Polymer and Carbon Materials, Polish Academy of Sciences, M. Curie-Sklodowska 34, 41-819 Zabrze
}

Received November 29, 2009; accepted December 11, 2009; published December 31, 2009

\begin{abstract}
A guest-host polymer system was formed from poly(methyl methacrylate) (PMMA) and the synthesized N-ethyl-N-(2hydroxylethyl)-4-(2-cyano-4-nitrophenylazo)aniline azobenzene chromophore. Linear optical properties have been determined for thin polymer films prepared by a spin-coating method. For the film poled in a moderate electric field the value of electro-optic (E-O) coefficient was measured by a simple reflection technique proposed by Teng and Man.
\end{abstract}

Organic materials are recognized as alternatives to inorganic crystals for optoelectronic and photonic applications. In recent years the design and synthesis of nonlinear optical (NLO) polymers has been a very dynamic area of materials research. Features that make the polymers interesting are their potentially larger optical nonlinearities and lower dielectric constants in comparison to traditional inorganic materials, their sufficiently high laser damage threshold, mechanical stability and easier processability and compatibility with integrated circuit technology. A unique polymer characteristics is the possibility of tailoring its physical properties through the proper modification of its chemical structure. The challenge is to design and synthesize processable, thermally stable polymers of high optical quality and nolinearity [1-3].

Among the variety of NLO systems, polymers containing dyes such as azobenzene derivatives are the most extensively studied. Azobenzene containing materials offer the possibility of changing the molecular arrangement optically, due to multiple trans $\rightarrow$ cis $\rightarrow$ trans isomerization cycles. The resulting photo-induced birefringence has potential applications in the area of information processing, optical storage and switching [4,5]. Moreover, azo dye doped polymers are interesting and extensively investigated materials due to their electro-optic properties and arising promising applications in high-speed modulators. E-O polymers are composed of chromophores showing both large both dipole moment and first hyperpolarizability. The chromophores are built of the electron donor and electron acceptor groups separated by a $\pi$ - conjugated bridge, and are incorporated into a polymer mainly by either dissolution in a polymer host or by covalent bonding to the macromolecular chain. The $\pi$ electrons can be easily and quickly redistributed in the molecule by an applied electric field. The resulting microscopic NLO response is determined by the given chromophore structural units. However, since the orientational distribution of NLO chromophores in the polymer films is isotropic the macroscopic second-order nonlinearities may be obtained only if the chromophores become oriented noncentrosymmetrically in the polymer matrix. The required alignment is commonly achieved by electric field poling. The technique involves heating the polymer to a temperature near its glass-transition temperature, applying a large electric field and cooling down the film in the presence of an electric field in order to freeze the chromophore alignment. The final material nonlinear activity is strongly dependent on the efficiency of the poling process.

In this paper we present the results of the measurements of linear optical properties and preliminary E-O response of the polymer system obtained by the dissolution of N-ethyl-N-(2-hydroxylethyl)-4-(2-cyano-4nitrophenylazo)aniline chromophore in poly(methyl methacrylate) matrix. In this chromophore, cyano and nitro groups act as electron acceptors and [N-ethyl-N-2hydroxylethyl]-amino group acts as an electron donor. To the best of our knowledge, such a chromophore has not been yet used as a dopant for preparation of guest-host systems.

Chemicals and solvents were purchased from Aldrich and used as received. Azo compound that is, N-ethyl-N(2-hydroxylethyl)-4-(2-cyano-4-nitrophenylazo)aniline (denoted as AZ-1 chromophore) was prepared as reported in our previous papers [6]. The molecular structure of an azochromophore is shown in Fig.1.

\footnotetext{
*E-mail: annak@if.pw.edu.pl
} 
<smiles>CCN(CCO)c1ccc(/N=N/c2ccc([N+](=O)[O-])cc2C#N)cc1</smiles>

Fig.1 Molecular structure of the N-ethyl-N-(2-hydroxylethyl)-4-(2cyano-4-nitrophenylazo)aniline azochromophore.

The AZ-1 compound was prepared by the azo coupling of 2-cyan-4-nitroaniline with 2-(N-ethylanilino)ethanol, that is, 2-cyan-4-nitroaniline was converted to the corresponding diazonium salt, which was subjected to coupling reaction with 2-(N-ethylanilino)ethanol to give the final compound. AZ-1: UV-Vis (NMP): $\lambda_{\max }$ $=548 \mathrm{~nm}, \varepsilon=1.12 \times 10^{4}[1 / \mathrm{mol} \mathrm{cm}]$, m.p. $=136^{\circ} \mathrm{C}$.

The matrix material was PMMA with an average molecular weight of $12.4 \times 10^{4} \mathrm{~g} / \mathrm{mol}$ and glass transition temperature $\mathrm{T}_{\mathrm{g}}=126^{\circ} \mathrm{C}$. Chromophore-doped PMMA film samples (10 wt.\%) were prepared in the following way: a chromophore and PMMA mixture was dissolved in chloroform and the resulting solution was filtered through a $0.2 \mu \mathrm{m}$ millipore filter. Very little residuum indicated that chromophores and PMMA had good compatibility. The films were fabricated by the spin-coating method followed by drying at $50^{\circ} \mathrm{C}$ for $3 \mathrm{~h}$ and at $90^{\circ} \mathrm{C}$ for $1 \mathrm{~h}$ in a vacuum oven. Two types of films were prepared, namely films on glass substrates for linear optical measurements and on a glass substrate precoated with an approximately 130-nm-thick indium tin oxide (ITO) layer for E-O measurements. UV-vis absorption and reflection spectra of films cast on glass substrates were recorded using a Jasco V570 UV-V-NIR spectrometer. The glass transition temperature of the chromophore-doped PMMA film was investigated by differential scanning calorimetry (DSC) measurements using a Du Pont 1090B apparatus with a heating rate of $20^{\circ} \mathrm{C} / \mathrm{min}$ under the nitrogen atmosphere.

For E-O measurement a 100 -nm-thick aluminum layer was sputter deposited on a $2.7-\mu \mathrm{m}$-thick polymer film. The ITO and aluminum layers served as poling and modulation electrodes. The poling was performed at $95^{\circ} \mathrm{C}$ in a moderate electric field of the order of $65 \mathrm{~V} / \mu \mathrm{m}$ for 10 minutes. The linear electro-optic response was examined using a reflection technique proposed by Teng and Man [7], in the experimental setup presented in Fig. 2.

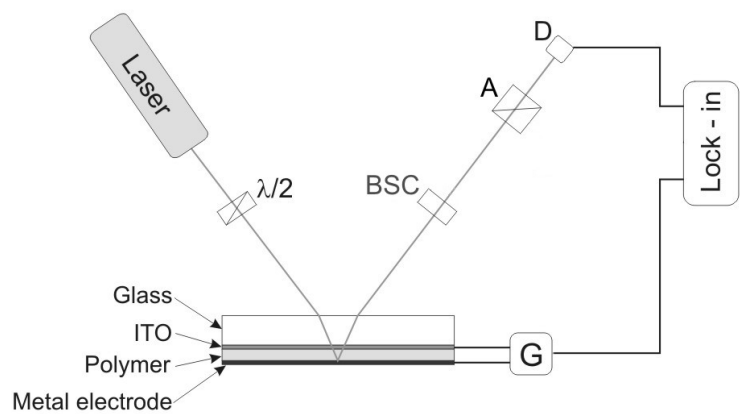

Fig.2. Experimental setup for E-O coefficient measurement; $\lambda / 2-$ halfwave plate, BSC - Babinet-Soleil compensator, A -analyzer, D detector, $\mathrm{G}$ - generator.

The measurement was performed in a crossed-polarizer configuration at a wavelength of $790 \mathrm{~nm}$, which is above the absorption band. A half-wave plate rotated vertical polarization of the laser beam by $45^{\circ}$ in order to equate the intensities of $\mathrm{s}$ and $\mathrm{p}$ orthogonal waves. A photodetector and a lock-in amplifier were used to detect light intensity modulations induced by a $1 \mathrm{kHz}$ ac voltage applied to the sandwiched structure. A Babinet-Soleil compensator was applied to adjust the relative mismatch between $\mathrm{s}$ and $\mathrm{p}$ components to get the maximum effect. The $r_{33}$ E-O coefficient of the poled polymer film was evaluated using the equation below [7]:

$$
r_{3}=\frac{3 \lambda I_{m}}{4 \pi V_{m} I_{c} n^{2}} \frac{\left(n^{2}-\mathrm{s}^{2} \mathrm{i} \theta\right) \mathrm{n}^{3 / 2}}{\left(n^{2}-2 \mathrm{~s}^{2} \mathrm{i} \theta\right) \mathrm{n} \mathrm{s}^{2} \mathrm{i} \theta}
$$

where $\lambda$ is the laser beam wavelength, $\theta$ is the angle of incidence, $n$ is the refractive index of the polymer, $I_{c}$ is the intensity at the photodetector, $V_{m}$ is the modulating voltage and $I_{m}$ is the modulating intensity.

The optical absorption spectrum of the prepared film sample is presented in Fig.3. The spectrum showed a strong band with its maximum located at ca $500 \mathrm{~nm}$ attributed to the electronic $\pi \rightarrow \pi^{*}$ and $n \rightarrow \pi^{*}$ transition of the trans-azo chromophore [8].

The refractive index was estimated at $1.75( \pm 0.02)$ from the Kramers-Kronig analysis applied to the reflectance spectra of the investigated film [9, 10]. The introduction of chromophores into a PMMA matrix resulted in decreasing the glass transition temperature of the PMMA/AZ-1 system to $110^{\circ} \mathrm{C}$. The $r_{33}$ E-O coefficient of the poled sample was estimated on 1.5 $\mathrm{pm} / \mathrm{V}$. 


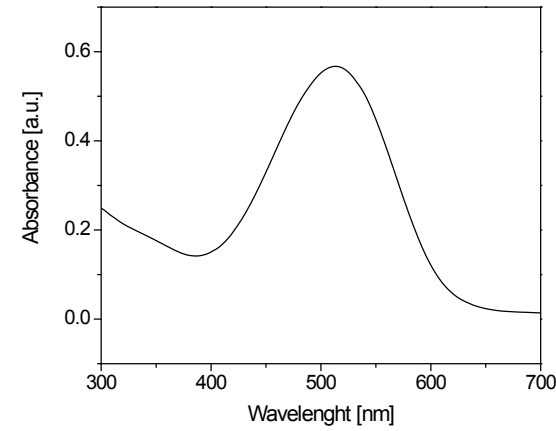

Fig.3. UV-Vis absorption spectrum of PMMA/AZ-1 system.

The initially measured value of $r_{33}$ coefficient of AZ-1 chromophore is going to be optimized further since, as mentioned, it is highly dependent on the efficiency of the poling procedure: the poling field and the poling temperature. The latest studies showed the E-O coefficient may be significantly increased in binary chromophore- containing polymers made of free chromophores dissolved in side-chain E-O polymer [11]. Thus, evaluating the final
E-O activity of the PMMA/AZ-1 system and examining its possibility of forming a binary system will be the subject of our future studies.

\section{References}

[1] H. Jiang, A.K. Kakkar, Macromolecules 31, 4170 (1998)

[2] S. K. Yesodha, Ch. K. S. Pillai and N. Tsutsumi, Prog. Polym. Sci. 29, 45 (2004).

[3] H.S. Nalwa, S. Miyata, Nonlinear Optics of Organic Molecules and Polymers (CRC Press. Inc., New York, 1997).

[4] L. Nikolova, P. Markovaky, V. Dragostinova, N. Mateva, Mod. Opt.35 (1988) 1789.

[5] T. Xu , G. Chen, C. Zhang, Z. Hao, X. Xu, J. Tian, Optical Materials 30, 1349 (2008).

[6] E. Schab-Balcerzak, M. Siwy, M. Kawalec, A. Sobolewska, A. Chamera, A. Miniewicz, J. Phys. Chem. A 113, 8765 (2009).

[7] C.C. Teng and H.T. Man, Appl. Phys. Lett. 56, 1734 (1990).

[8] H. Rau. In Photochemistry and Photophysics, F.J. Rabek Ed., CRC, Boca Raton, FL, 1990, Vol. II, Chapter 4, pp 119-141.

[9] J. Fellman, T. Westerlund, J. Non-Cryst. Solids 146, 165 (1992).

[10] B. Jarzabek, J. Weszka, M. Domanski, J. Jurusik, J. Cisowski, J. Non-Cryst. Solids 354, 856 (2008).

[11] Y.V. Pereverzev, K.N. Gunnerson, O.V. Prezhdo, P.A. Sullivan, Y. Liao, B.C. Olbricht, A.J. Akelaitis, A.K.-Y. Jen, L.R. Dalton, J. Phys. Chem. C 112, 4355 (2008). 